\title{
The effects of preoperative discontinuation of antithrombotic medications on the risks for thrombosis and bleeding in patients undergoing thoracic surgery
}

\author{
Ayten Saracoglu', Didem Güngör ${ }^{2}$, Esra Şirazi², Bedrettin Yıldızeli², \\ Mustafa Yüksel'2, Zuhal Aykaç' \\ Department of Anesthesiology and Intensive Care', Thoracic Surgery², Marmara \\ University Medical School, Istanbul, Turkey
}

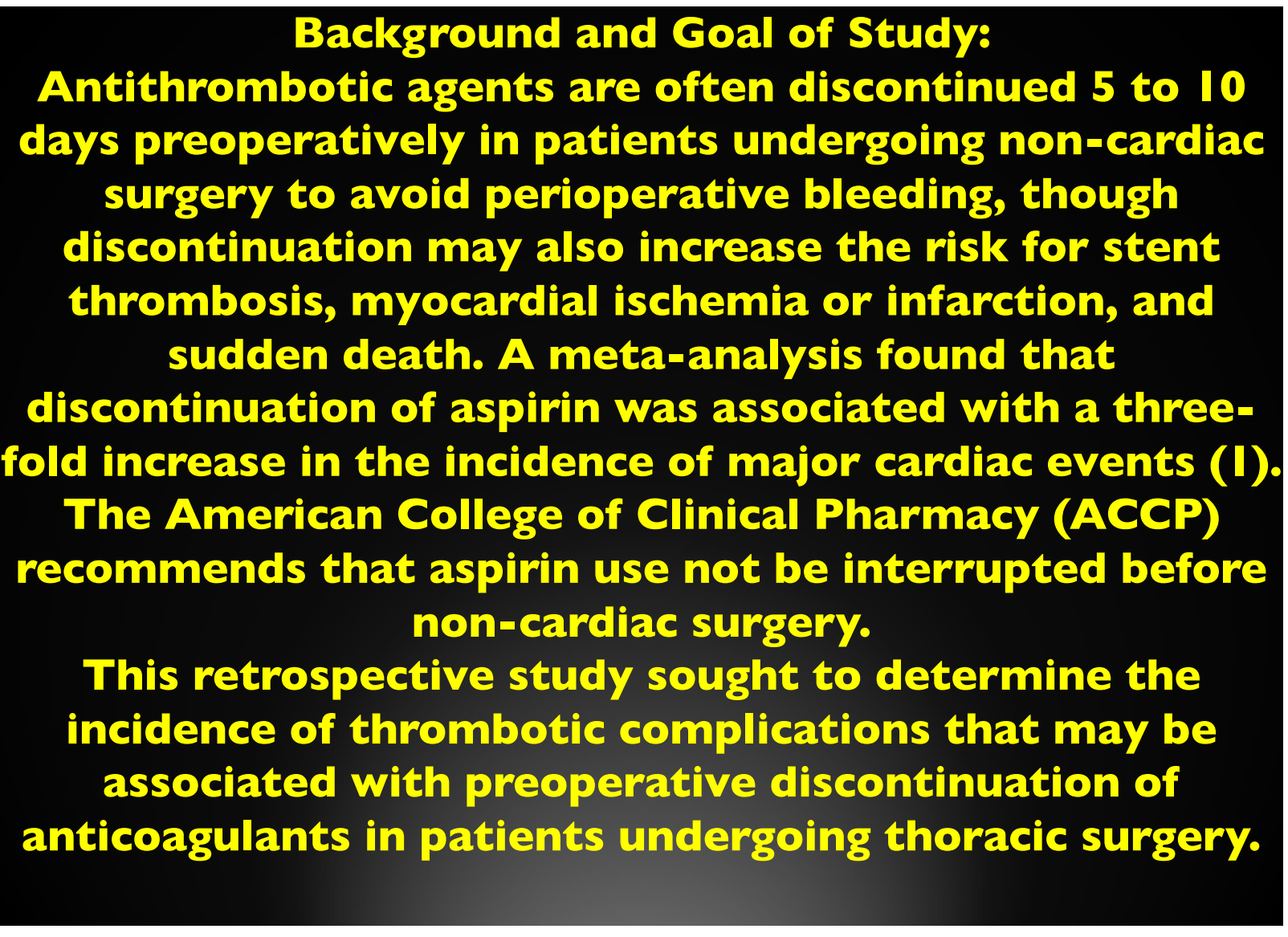

After obtaining the approval of the institutional review board, the study included I 26 patients who underwent thoracic surgery between January 2014 and June 2017. The patients were divided into two groups based on no medication and discontinuation of anticoagulant agents prior to surgery. Data were collected on demographic features, cardiac reserves, pre- and postoperative

hemograms and other laboratory tests, the frequency, duration and type of anticoagulants used, type of thoracic surgery, the amount of blood and blood products transfused, the need for re-exploration, length of hospital and ICU stay, complications, and mortality.

\section{Results:}

There were no significant differences between the two groups in demographic characteristics, length of hospital and ICU stay, levels of Hb, Htc, and creatinin, the amount of perioperative fluid, platelet suspension, the use of ES, postoperative events (DVT, MI, and stroke), and the rates of severe arrhythmias, re-exploration, and mortality ( $p>0,05$, Table I). Postoperative DVT and MI incidences were $2.9 \%$ in patients with discontinued antithrombotic agents. Patients who discontinued antithrombotic medication had a significantly higher preoperative INR value than no medication group $(p<0,05)$. able 1: Patient demographics, transfusion rates and complications

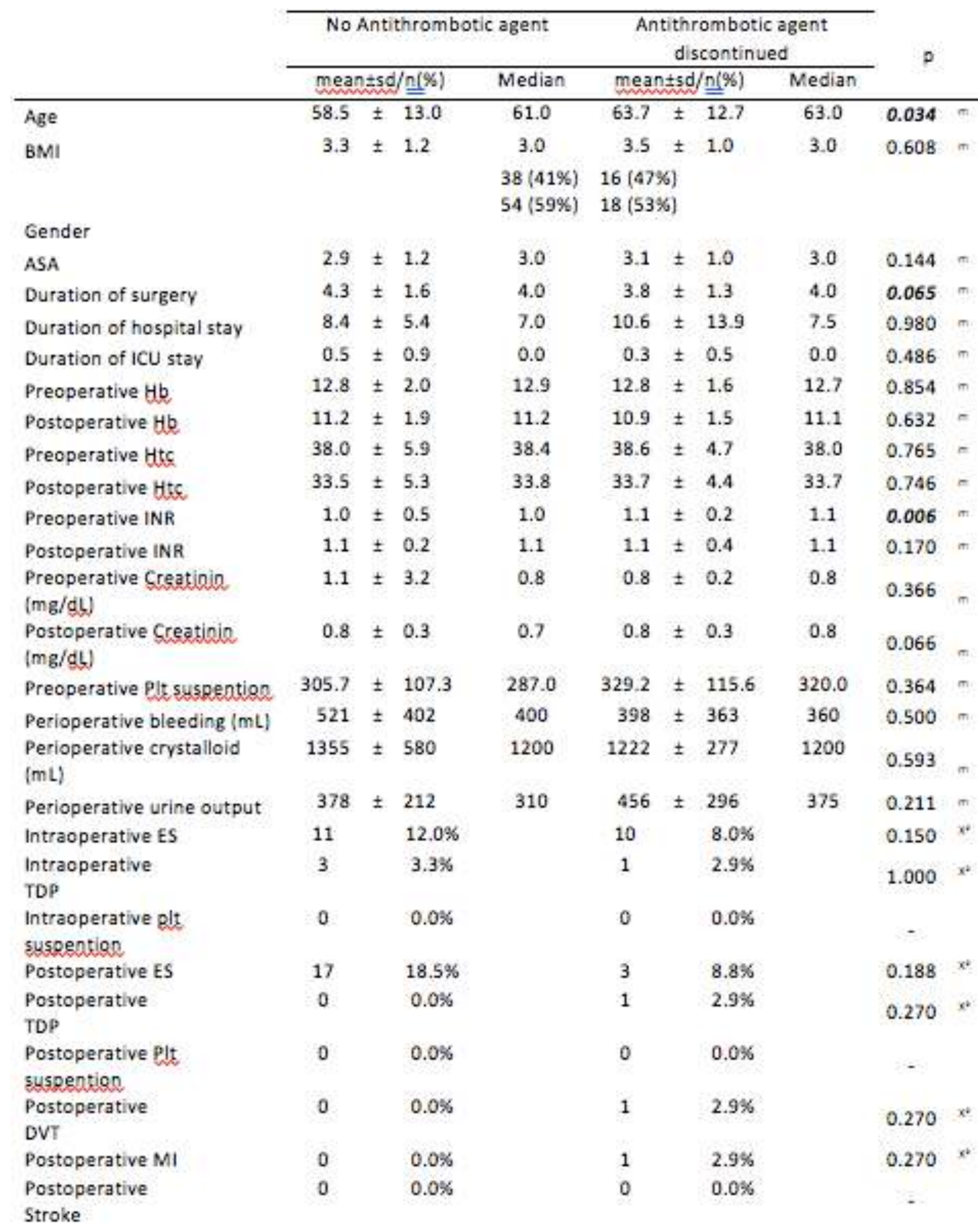

Although there was no statistically significant difference between groups, it seems that the discontinuation of antithrombotic agents may be related with thrombotic serious complications in patients undergoing thoracic surgery. 